\title{
Within-day and between-days reproducibility of isokinetic parameters of knee, trunk and shoulder movements
}

\author{
Sam D. Blacker ${ }^{\mathrm{a}}$, Joanne L. Fallowfield ${ }^{\mathrm{b}}$, James L.J. Bilzon ${ }^{\mathrm{c}}$ and Mark E.T. Willems ${ }^{\mathrm{a}, *}$ \\ ${ }^{a}$ Faculty of Sport, Education \& Social Sciences, University of Chichester, Chichester, West Sussex, UK \\ ${ }^{\mathrm{b}}$ Institute of Naval Medicine, Gosport, Hampshire, UK \\ ${ }^{\mathrm{c}}$ School for Health, University of Bath, Bath, UK
}

\begin{abstract}
The purpose of this study was to examine the within-day and between-days reproducibility of isokinetic parameters of knee, shoulder and trunk movements. Ten healthy males (age $30 \pm 8$ years, height $1.79 \pm 0.05 \mathrm{~m}$, body mass $79.4 \pm 8.3 \mathrm{~kg}$ ) performed isokinetic concentric contractions (knee: 60 and $180^{\circ} / \mathrm{s}$; shoulder: 60 and $180^{\circ} / \mathrm{s}$ : and; trunk: 15 and $60^{\circ} / \mathrm{s}$ ) at 0 (baseline), 2, 24, 48 and 72 hours. Reproducibility of the baseline peak moment, mean peak moment and maximum work was assessed using Bland and Altman limits of agreement and pre-planned paired t-tests $(P<0.05)$. Peak moment of knee, trunk and shoulder extensors at low velocities had highest reproducibility within and between days over a time period of 3 days. Peak moment at high and low velocities of knee extensors was consistently the most reliable parameter over a 3 day period. It is concluded that isokinetic parameters can be reproducibly measured in a combined session of knee, shoulder and trunk movements in healthy male subjects over a 3-day time period.
\end{abstract}

Keywords: Dynamometry, reproducibility, torque, extension, flexion, muscle

\section{Introduction}

The quadriceps femoris is a muscle group that allows easy access and is therefore frequently the focus of studies assessing neuromuscular impairment following exercise $[4,23,25,27]$. However, dynamic whole body exercise such as load carriage, requires the use of a range of muscle groups. Overuse of these muscle groups may result in muscle damage and therefore a reduction in muscle function [1]. This may have adverse neuromuscular [11] and metabolic [37] consequences. More important, it can impact on occupational performance in the days following an exercise bout. However, it is not known whether testing of knee, trunk and shoulder movements for healthy subjects in one session is reproducible over a period of days.

${ }^{*}$ Corresponding author: Dr. Mark Willems, Faculty of Sport, Education \& Social Sciences, University of Chichester, Chichester, PO19 6PE West Sussex, UK. Tel.: +44 (0)1243 816468; Fax: +44 (0)1243 816080; E-mail: m.willems@chi.ac.uk.
Isokinetic dynamometry has been used to assess the decreases and recovery of muscle torque following activities known to cause muscle damage [16,29,31]. A typical method is to measure the maximal amount of torque or work a muscle group can produce before the activity with recovery measured in the following hours and days [10,16,19]. Knapik and Ramos [22] suggested that with different isokinetic test velocities motor tasks become more dissimilar, requiring different patterns of neural recruitment and co-ordination. Therefore multiple test velocities are commonly used to assess muscle function under a range of different conditions.

The reproducibility of isokinetic dynamometer testing has been previously assessed for measururements of extensors and flexors of the knees [20,35], trunk [9, $14,24]$ and shoulders [26,30]. These studies have assessed both intra and interday reproducibility normally using a test and single re-test design or a multiple re-test design. However, the reproducibility of the measurements of the knee, trunk and shoulder extensors and 
flexors in one testing session within-day and between days has not been examined.

Therefore, the aim of this study was to examine the reproducibility of concentric contractions of extensors and flexors of the knee, trunk and shoulder tested in one session with isokinetic dynamometry over a 3-day period. This study may have implications for studies on the functional recovery from exercise that may result in injury of multiple muscle groups such as prolonged load carriage as it common in certain occupational settings. Our study addressed the question whether the testing of knee, trunk and shoulder movements in one session is reproducible over a period of three days.

\section{Methods}

\subsection{Participants}

Ten healthy male participants (mean \pm SD, age 30 \pm 8 years, height $1.79 \pm 0.05 \mathrm{~m}$, body mass $79.4 \pm$ $8.3 \mathrm{~kg}$ ) volunteered to participate in this study. Ethical approval for all procedures and protocols was provided by the University of Chichester Ethics Committee. All protocols were performed in accordance with the ethical standards laid down in the 1964 Declaration of Helsinki. Participants provided written informed consent and were free from any musculoskeletal injury prior to commencing the study.

Participants were instructed to abstain from any vigorous and unaccustomed physical activity 24 hours before and for the duration of the study to avoid muscle damage and arrive in the laboratory in a rested state. Participants were physically active but not engaged in formal training with the muscle groups of interest. Before baseline testing participants were questioned to ensure they were adequately rested and free from muscle injury and completed a muscle soreness questionnaire. The questionnaire provided a map of the body divided into 12 segments [13]; the perception of muscle soreness in each segment was rated on a visual analogue scale from 0 (no soreness) to 10 (unbelievable soreness). All participants reported the lowest rating of 0 (no soreness) for all body segments before each baseline testing session.

\subsection{Pre-testing}

Subject's body mass (Seca Model 880, Seca Ltd., Birmingham, UK) was taken whilst wearing shorts and underwear and stature recorded (Avery Berkel, Smethwick, UK).

At least 5 days prior to beginning the experimental protocol, participants were familiarised with all test procedures by completing one complete cycle of the experimental protocol (described in detail below). A test procedure was repeated if a maximal effort was not given or a learning effect was still apparent in the final contractions (i.e. peak force was greater during the final compared to the penultimate contraction).

\subsection{Experimental protocol}

Subjects completed the muscle function tests described below at 0 (baseline), 2, 24, 48 and 72 hours to assess reproducibility of test procedures. The test order is described below and was kept the same on each occasion (Fig. 1). The test protocol was designed to measure the force producing capability of the muscles. The number of sets and repetitions of contractions was based on pilot work in our laboratory. Rest periods were enforced to minimise fatigue and allow time for participants to move between dynamometers. During all tests, standard verbal encouragement was provided [21]. Slow to fast test velocities were chosen (details described below) as there are known variations in motor unit recruitment patterns and muscle fibre composition between individuals and between muscle groups in any one individual [33].

Knee and shoulder extension and flexion data were recorded (Cybex II isokinetic dynamometer, Cybex, Measham, UK) using HUMan Assessment Computer (HUMAC) software V40 (Computer Sports Medicine Inc, Norwood, USA) at $100 \mathrm{~Hz}$ and exported to Microsoft Excel 2002 for Windows (Microsoft, Redmond, Washington) for analysis. Data were corrected for the effect of gravity [17]. Trunk extension and flexion data were recorded (Akron Isokinetic Back System, Akron Therapy Products, Ipswich, UK) at $100 \mathrm{~Hz}$ using Akron software V2.4 (Akron Therapy Products, Ipswich, UK) and exported for analysis in Microsoft Excel 2002 for Windows (Microsoft, Redmond, Washington). Data were not corrected for the effect of gravity due to the limitations of the dynamometer. Although this is not considered best practice [21], as data were not corrected in each session, the test-retest reproducibility can still be accurately assessed. Caution would be required if extension/flexion ratios were calculated or if comparisons of parameters (e.g. peak torque, work done) are made with data which has been corrected for the effect of gravity. 


\begin{tabular}{|c|c|c|c|c|c|c|}
\hline \multicolumn{7}{|c|}{ Isokinetic Trunk Extension and Flexion } \\
\hline & & $3 \times 15^{\circ} / \mathrm{s}$ & $30 \mathrm{~s}$ Rest & $3 \times 60 \% / s$ & & \\
\hline \multicolumn{7}{|c|}{180 s Rest } \\
\hline \multicolumn{7}{|c|}{ Isokinetic Knee Extension and Flexion } \\
\hline $5 \times 60^{\circ} / \mathrm{s}$ & 10 s Rest & $5 \times 60^{\circ} / \mathrm{s}$ & 30 s Rest & $5 \times 180^{\circ} / \mathrm{s}$ & 10 s Rest & $5 \times 180^{\circ} / \mathrm{s}$ \\
\hline \multicolumn{7}{|c|}{180 s Rest } \\
\hline \multicolumn{7}{|c|}{ Isokinetic Shoulder Extension and Flexion } \\
\hline $5 \times 60^{\circ} / \mathrm{s}$ & $10 \mathrm{~s}$ Rest & $5 \times 60^{\circ} / \mathrm{s}$ & 30 s Rest & $5 \times 180^{\circ} / \mathrm{s}$ & 10 s Rest & $5 \times 180^{\circ} / \mathrm{s}$ \\
\hline
\end{tabular}

Fig. 1. Schematic of test battery to measure dynamic isokinetic contractions of the trunk $\left(15\right.$ and $\left.60^{\circ} / \mathrm{s}\right)$, knee $\left(60\right.$ and $\left.180^{\circ} / \mathrm{s}\right)$ and shoulder $(60$ and $180^{\circ} / \mathrm{s}$ ) extensors and flexors at 0 (baseline), 2, 24, 48 and $72 \mathrm{~h}$.

Slower test velocities were tested first for all isokinetic contractions to increase reproducibility of results between tests [41]. For all contractions, the angular velocity was calculated every 0.01 seconds during the movement and data were removed if they were not collected during the isokinetic phase of the movement or showed torque overshoot [33].

\subsection{Isokinetic knee extension and flexion}

Subjects were seated in the test chair of a Cybex II isokinetic dynamometer (Cybex, Measham, UK) with their knee at $90^{\circ}$ flexion secured using a seat belt style strap across their chest and hips. The Cybex long input adapter, adjustable arm and shin pad (Cybex, Measham, UK) were attached to the dynamometer's point of rotation and to the ankle of the non-dominant leg via a Velcro cuff as instructed in the Cybex user manual. The dominant leg was placed behind the restraining bar to prevent movement during measurement. The point of rotation of the dynamometer arm was aligned, using a laser pointer, with the lateral femoral epicondyle [15]. Subject range of motion was restricted by mechanical stops at $70^{\circ}$ (flexion) and $0^{\circ}$ (extension) of the knee to prevent hyper extension or flexion. Three sub-maximal (self-perceived 50\% effort) contractions (full extension and flexion) were completed to familiarise participants to the experimental set up in each test session. The test protocol consisted of two sets of five maximal dynamic contractions of the knee extensors and flexors at 60 and $180^{\circ} / \mathrm{s}$, each separated by $30 \mathrm{~s}$ rest (Fig. 1 ).

\subsection{Isokinetic trunk extension and flexion}

Subjects were positioned standing upright (trunk fully extended, $0^{\circ}$ ) in an isokinetic trunk strength dy- namometer (Akron Therapy Products, Ipswich, UK). Movement was restricted to the use of the abdominal and back muscles only between extension $\left(5^{\circ}\right)$ and flexion $\left(50^{\circ}\right)$ of the start position. Straps were placed across the subject's upper and lower legs and hips and a frame positioned around the shoulders. The point of rotation of the dynamometer was aligned with the L5-S1 vertebrae, using a laser pointer [15]. This was located by identifying the highest point of the iliac crest and measuring $5 \mathrm{~cm}$ dorsally and $5 \mathrm{~cm}$ laterally from this point. Mechanical stops were applied at $5^{\circ}$ and $50^{\circ}$ of the subject's vertical position to limit their range of movement and prevent hyper extension or flexion. Three sub-maximal (self-perceived 50\% effort) contractions (full extension and flexion) were completed to familiarise participants to the experimental set up in each test session. The test protocol consisted of two sets of three maximal dynamic contractions of the trunk extensors and flexors at 15 and $60 \%$ s, each separated by $30 \mathrm{~s}$ rest (Fig. 1).

\subsection{Isokinetic shoulder extension and flexion}

Subjects lay in a supine position on a custom made testing couch placed parallel to a Cybex II isokinetic dynamometer (Cybex, Measham, UK). The Cybex offset input adapter, shoulder testing accessory and neutral handgrip (Cybex, Measham, UK) were attached to the dynamometer. Subjects gripped the handle in their right hand; the adapter length was adjusted so their right arm was fully extended $\left(0^{\circ}\right)$ (i.e. minimal flexion in the elbow). Subject's movement was restricted by securing Velcro straps across the upper legs and hips with the left arm placed across the chest. The point of rotation of the dynamometer arm was aligned with the right 
Acromiale using a laser pointer [15]. Due to laboratory restrictions subjects were tested on their right arm only, but very little difference in strength exists between dominant and non- dominant arms for flexion (0.9 to $1.2 \mathrm{Nm}$ ) or extension $(0.1$ to $0.9 \mathrm{Nm})$ [33]. Mechanical stops were placed the end of the range of movements to prevent hyper extension and flexion. Range of motion was between $0^{\circ}$ and $180^{\circ}$. Three sub-maximal (selfperceived $50 \%$ effort) contractions (full extension and flexion) were completed to familiarise participants to the experimental set up in each test session. The test protocol consisted of two sets of five maximal dynamic contractions of the shoulder extensors and flexors at 60 and $180^{\circ} / \mathrm{s}$, each separated by $30 \mathrm{~s}$ rest (Fig. 1).

\subsection{Calculated variables}

The following variables were calculated for all maximal dynamic contractions [32], after removal of data not collected during the isokinetic phase $(<1 \%$ of all data) and correction for gravity (knee and shoulder only): (a) peak moment, the single highest moment value recorded in all contractions at the specified speed (of the 10 contractions for knee and shoulder or 3 contractions for trunk); (b) mean peak moment, the mean of all maximum moment values recorded in all contractions at the specified speed; (c) maximum work done, the area under the curve of each contraction was calculated and the maximum value was reported.

\subsection{Environmental conditions}

Environmental temperature was monitored using a dry bulb thermometer (Fisher Scientific, Loughborough, UK). No differences in environmental temperature were observed between baseline tests at 2, 24, 48 and 72 hour test periods respectively $(21.2 \pm 2.5,22.2$ $\pm 2.6,21.6 \pm 1.0,21.6 \pm 1.5^{\circ} \mathrm{C}, 22.3 \pm 1.9^{\circ} \mathrm{C}, P>$ $0.05)$.

\subsection{Statistical analysis}

Statistical analysis was undertaken using SPSS for Windows V15 (SPSS, Chicago, Illinois) and Microsoft Excel 2002 for Windows (Microsoft, Redmond, Washington). Normal distribution of the data was verified using a Kolmogorov-Smirnov test.

Reproducibility of the baseline contractile parameters and measurements were assessed at 2, 24, 48 and 72 hours after baseline using Bland and Altman's $95 \%$ Limits of Agreement (LoA) [5-8]. Studies on the re- producibility of isokinetic dynamometry have used primarily correlation methods (e.g. Pearsons r, intraclass correlation coefficients) [33]. However, such methods have limitations [3] as they cannot detect systematic bias, are dependent on the range of values in a sample (i.e. a decrease in the spread of the data decreases the correlation therefore reproducibility), and are therefore only a measure of 'relative reproducibility'. However, these limitations are not present when using the LoA method which provides a measure of absolute reproducibility [3].

Data were examined for heteroscedasticity using Pearsons Correlation Coefficient. Heteroscedasticity was present in the majority of comparisons (as is the case for the majority of variables measured on the ratio scale), therefore, log ratio LoA were calculated and used for analysis of all data sets [3,28]. Logarithms for each data point were calculated and individual subject differences [baseline - test (2, 24, 48, 72 hours)] were plotted against respective individual means. Mean differences (bias) between baseline at 2, 24, 48, and 72 hour tests were assessed using pre-planned paired T-tests with statistical significance set at $P<0.05$. The 95\% LoA were produced by calculating the standard deviation of the differences between tests at different time points and multiplied by 1.96 .

\section{Results}

Absolute values of peak moment, mean peak moment and maximum work at 0 (baseline), 2, 24, 48 and 72 hours are presented in Tables $1-3$. In Tables $4-6$ are presented reproducibility of peak moment, mean peak moment and maximum work between 0 hours (baseline) and 2, 24, 48 and 72 hours.

\subsection{Knee extension $\left(60^{\circ} / \mathrm{s}\right)$}

There was no significant bias at 2, 24, 48 and 72 hours for the maximum and mean peak moment (Table 4). For maximum work, there was only a significant bias at 48 hours $(5.9, P=0.041)$. Reproducibility (as shown by $95 \%$ LoA) was similar for the maximum and mean peak moment but was consistently higher for maximum work at all time points (Table 4). Figure 2A provides an illustration of the bias and 95\% LoA for the mean peak moment knee extension at $60 \%$ s at baseline vs. 2 hours showing strong agreement between mean peak moment measurements with non-significant bias. 

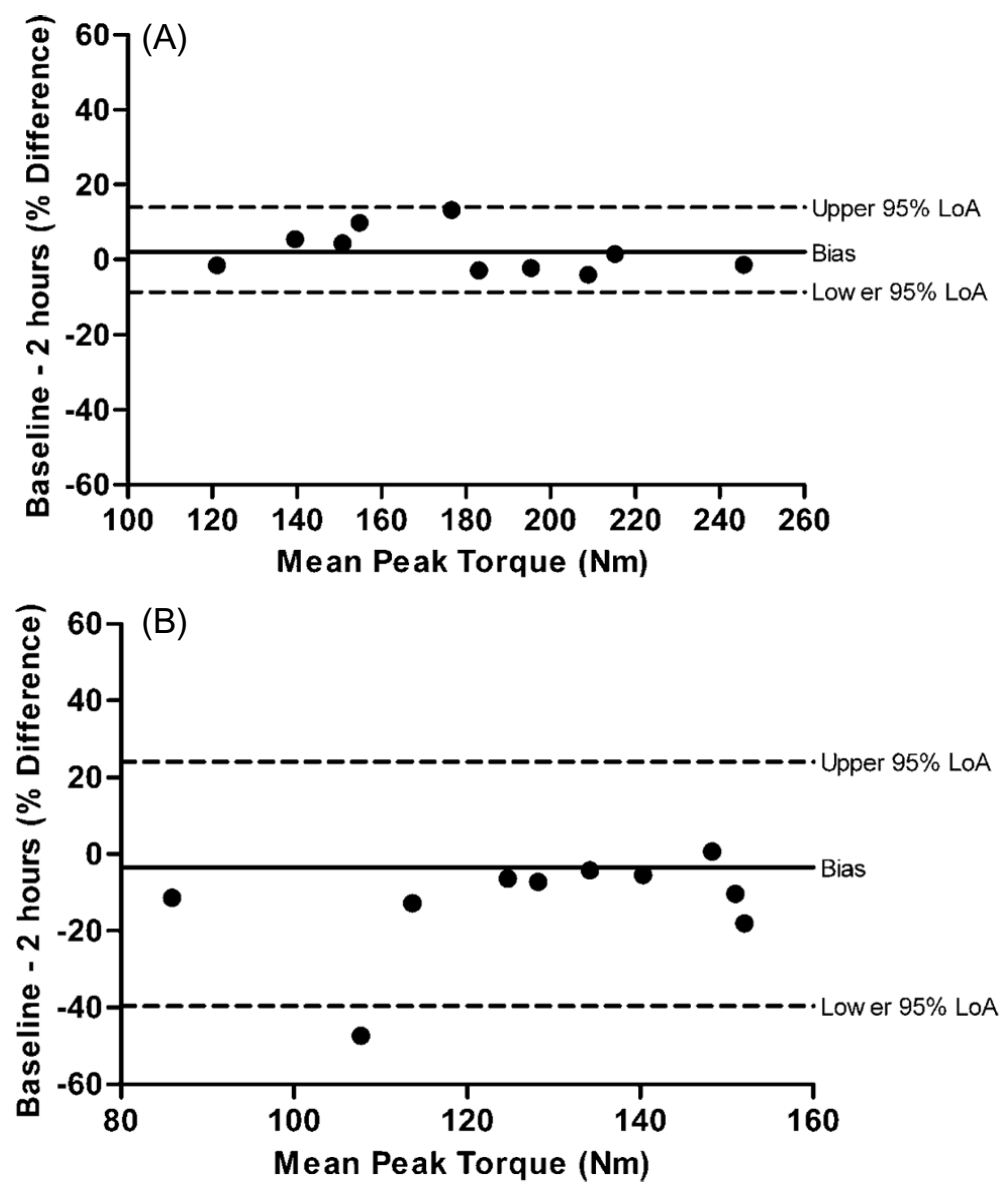

Fig. 2. Bland-Altman plots showing the agreement between mean peak moment at baseline ( 0 Hours) and 2 hours for knee extension (A) $60^{\circ} / \mathrm{s}$ (B) knee extension $180^{\circ} / \mathrm{s}$. Each data point is presented individually (mean baseline and 2 hour values vs. percentage differences between baseline and 2 hour values). The mean bias (-), 95\% limits of agreement (- - ) are displayed.

\subsection{Knee extension $\left(180^{\circ} / \mathrm{s}\right)$}

The bias for the peak moment at 2, 24, 48 and 72 hours (Table 4) was not significant. For peak moment, the reproducibility (as shown by $95 \%$ LoA) was typically stronger at all time points compared to the mean peak moment and maximum work (Table 4). For example, at 72 hours, the $95 \%$ LoA was 13.3 for the peak moment, 25.6 for the mean peak moment and 40.3 for the maximum work. Figure $2 \mathrm{~B}$ provided an illustration of the bias and 95\% LoA for the mean peak moment knee extension at $180 \%$ s at baseline vs. 2 hours showing weaker agreement between peak torque measurements with a significant negative bias.

\subsection{Knee flexion $\left(60^{\circ} / \mathrm{s}\right)$}

At 2, 24, 48 and 72 hours there was a non-significant bias for the peak moment, mean peak moment and max- imum work. The 95\% LoA was similar for peak moment and mean peak moment but higher for maximum work (Table 4).

\subsection{Knee flexion $\left(180^{\circ} / \mathrm{s}\right)$}

There was significant positive bias for the peak moment at $2(3.7, P=0.031)$ and $24(6.4, P=0.047)$ hours, and maximum work at 48 hours $(13.3, P=$ 0.047). For all parameters the reproducibility $(95 \%$ LoA) was similar at 2, 24, 48 and 72 hours (Table 4).

\subsection{Trunk extension $\left(15^{\circ} / \mathrm{s}\right)$}

There was non-significant bias at 2, 24, 48 and 72 hours for peak moment, mean peak moment and maximum work (Table 5). Although there was variation in the $95 \%$ LoA across time points, no obvious 
Table 1

Peak moment, mean peak moment and maximum work during isokinetic contractions of the knee extensors and flexors at test velocities of 60 and $180^{\circ} / \mathrm{s}(n=10)$ at 0 (baseline) 2, 24, 48 and 72 hours. Data are presented as mean \pm SD

\begin{tabular}{|c|c|c|c|c|c|c|}
\hline Measurement & & Baseline $(0 \mathrm{~h})$ & $2 \mathrm{~h}$ & $24 \mathrm{~h}$ & $48 \mathrm{~h}$ & $72 \mathrm{~h}$ \\
\hline \multirow[t]{3}{*}{ Knee Extension $\left(60^{\circ} / \mathrm{s}\right)$} & Peak Moment (Nm) & $198 \pm 40$ & $199 \pm 40$ & $197 \pm 44$ & $197 \pm 40$ & $202 \pm 40$ \\
\hline & Mean Peak Moment (Nm) & $178 \pm 40$ & $181 \pm 37$ & $179 \pm 42$ & $183 \pm 38$ & $184 \pm 37$ \\
\hline & Maximum Work (J) & $143 \pm 34$ & $148 \pm 32$ & $146 \pm 32$ & $151 \pm 32$ & $150 \pm 33$ \\
\hline \multirow[t]{3}{*}{ Knee Extension $\left(180^{\circ} / \mathrm{s}\right)$} & Peak Moment (Nm) & $142 \pm 28$ & $142 \pm 28$ & $143 \pm 25$ & $143 \pm 27$ & $145 \pm 28$ \\
\hline & Mean Peak Moment (Nm) & $112 \pm 27$ & $111 \pm 26$ & $115 \pm 21$ & $115 \pm 25$ & $117 \pm 24$ \\
\hline & Maximum Work (J) & $129 \pm 28$ & $129 \pm 27$ & $132 \pm 25$ & $132 \pm 27$ & $132 \pm 26$ \\
\hline \multirow[t]{3}{*}{ Knee Flexion $\left(60^{\circ} / \mathrm{s}\right)$} & Peak Moment (Nm) & $125 \pm 24$ & $125 \pm 20$ & $126 \pm 21$ & $128 \pm 20$ & $125 \pm 19$ \\
\hline & Mean Peak Moment (Nm) & $110 \pm 21$ & $113 \pm 16$ & $113 \pm 19$ & $115 \pm 18$ & $112 \pm 20$ \\
\hline & Maximum Work (J) & $105 \pm 21$ & $107 \pm 17$ & $107 \pm 18$ & $109 \pm 16$ & $107 \pm 17$ \\
\hline \multirow[t]{3}{*}{ Knee Flexion $\left(180^{\circ} / \mathrm{s}\right)$} & Peak Moment (Nm) & $93 \pm 13$ & $96 \pm 12$ & $99 \pm 14$ & $98 \pm 17$ & $98 \pm 16$ \\
\hline & Mean Peak Moment (Nm) & $83 \pm 13$ & $85 \pm 11$ & $87 \pm 12$ & $87 \pm 16$ & $88 \pm 17$ \\
\hline & Maximum Work $(\mathrm{J})$ & $20 \pm 5$ & $21 \pm 4$ & $22 \pm 5$ & $23 \pm 4$ & $22 \pm 5$ \\
\hline
\end{tabular}

Table 2

Peak moment, mean peak moment and maximum work during isokinetic contractions of the knee extensors and flexors at test velocities of 15 and $60^{\circ} / \mathrm{s}(n=8)$ at 0 (baseline) 2, 24, 48 and 72 hours. Data are presented as mean \pm SD

\begin{tabular}{|c|c|c|c|c|c|c|}
\hline Measurement & & Baseline $(0 \mathrm{~h})$ & $2 \mathrm{~h}$ & $24 \mathrm{~h}$ & $48 \mathrm{~h}$ & $72 \mathrm{~h}$ \\
\hline \multirow[t]{3}{*}{ Trunk Extension $\left(15^{\circ} / \mathrm{s}\right)$} & Peak Moment $(\mathrm{Nm})$ & $288 \pm 38$ & $285 \pm 51$ & $289 \pm 42$ & $278 \pm 48$ & $273 \pm 58$ \\
\hline & Mean Peak Moment (Nm) & $276 \pm 48$ & $273 \pm 50$ & $273 \pm 46$ & $265 \pm 54$ & $266 \pm 58$ \\
\hline & Maximum Work (J) & $1119 \pm 172$ & $1101 \pm 219$ & $1074 \pm 221$ & $1101 \pm 227$ & $1125 \pm 309$ \\
\hline \multirow[t]{3}{*}{ Trunk Extension $\left(60^{\circ} / \mathrm{s}\right)$} & Peak Moment (Nm) & $276 \pm 53$ & $272 \pm 51$ & $275 \pm 61$ & $277 \pm 60$ & $266 \pm 64$ \\
\hline & Mean Peak Moment (Nm) & $266 \pm 56$ & $264 \pm 53$ & $265 \pm 62$ & $270 \pm 62$ & $261 \pm 65$ \\
\hline & Maximum Work (J) & $215 \pm 58$ & $213 \pm 54$ & $198 \pm 44$ & $228 \pm 66$ & $192 \pm 59$ \\
\hline \multirow[t]{3}{*}{ Trunk Flexion $\left(15^{\circ} / \mathrm{s}\right)$} & Peak Moment (Nm) & $259 \pm 44$ & $258 \pm 44$ & $259 \pm 41$ & $257 \pm 45$ & $253 \pm 50$ \\
\hline & Mean Peak Moment (Nm) & $252 \pm 44$ & $250 \pm 44$ & $252 \pm 44$ & $247 \pm 49$ & $245 \pm 47$ \\
\hline & Maximum Work $(\mathrm{J})$ & $1010 \pm 247$ & $978 \pm 196$ & $961 \pm 187$ & $946 \pm 190$ & $1049 \pm 409$ \\
\hline \multirow[t]{3}{*}{ Trunk Flexion $\left(60^{\circ} / \mathrm{s}\right)$} & Peak Moment (Nm) & $296 \pm 34$ & $290 \pm 37$ & $291 \pm 34$ & $290 \pm 40$ & $299 \pm 39$ \\
\hline & Mean Peak Moment (Nm) & $287 \pm 37$ & $280 \pm 41$ & $283 \pm 37$ & $281 \pm 42$ & $288 \pm 49$ \\
\hline & Maximum Work $(\mathrm{J})$ & $263 \pm 63$ & $268 \pm 79$ & $257 \pm 69$ & $260 \pm 68$ & $273 \pm 71$ \\
\hline
\end{tabular}

Table 3

Peak moment, mean peak moment and maximum work during isokinetic contractions of the shoulder extensors and flexors at test velocities of 60 and $180^{\circ} / \mathrm{s}(n=10)$ at 0 (baseline) 2, 24, 48 and 72 hours. Data are presented as mean \pm SD

\begin{tabular}{|c|c|c|c|c|c|c|}
\hline Measurement & & Baseline $(0 \mathrm{~h})$ & $2 \mathrm{~h}$ & $24 \mathrm{~h}$ & $48 \mathrm{~h}$ & $72 \mathrm{~h}$ \\
\hline \multirow[t]{2}{*}{ Shoulder Extension $\left(60^{\circ} / \mathrm{s}\right)$} & Peak Moment (Nm) & $101 \pm 20$ & $101 \pm 18$ & $103 \pm 22$ & $106 \pm 26$ & $103 \pm 23$ \\
\hline & Mean Peak Moment (Nm) & $89 \pm 16$ & $90 \pm 17$ & $90 \pm 18$ & $92 \pm 20$ & $91 \pm 18$ \\
\hline \multirow[t]{3}{*}{ Shoulder Extension $\left(180^{\circ} / \mathrm{s}\right)$} & Peak Moment (Nm) & $85 \pm 15$ & $86 \pm 17$ & $83 \pm 15$ & $88 \pm 21$ & $87 \pm 17$ \\
\hline & Mean Peak Moment (Nm) & $76 \pm 13$ & $77 \pm 15$ & $75 \pm 14$ & $79 \pm 18$ & $77 \pm 14$ \\
\hline & Maximum Work (J) & $40 \pm 8$ & $42 \pm 9$ & $41 \pm 8$ & $42 \pm 10$ & $41 \pm 8$ \\
\hline Shoulder Flexion $\left(60^{\circ} / \mathrm{s}\right)$ & Maximum Work (J) & $114 \pm 21$ & $115 \pm 19$ & $116 \pm 22$ & $121 \pm 27$ & $112 \pm 22$ \\
\hline \multirow[t]{3}{*}{ Shoulder Flexion $\left(180^{\circ} / \mathrm{s}\right)$} & Peak Moment (Nm) & $54 \pm 9$ & $55 \pm 6$ & $56 \pm 8$ & $53 \pm 7$ & $55 \pm 7$ \\
\hline & Mean Peak Moment (Nm) & $46 \pm 9$ & $48 \pm 6$ & $48 \pm 6$ & $46 \pm 6$ & $48 \pm 6$ \\
\hline & Maximum Work $(\mathrm{J})$ & $23 \pm 5$ & $24 \pm 3$ & $25 \pm 4$ & $23 \pm 4$ & $24 \pm 4$ \\
\hline
\end{tabular}

trend was observed (Table 5). The variation was due to individual outlying data points for each variable which increase the 95\% LoA, such as that shown in Fig. 2B for knee extension.

\subsection{Trunk extension $\left(60^{\circ} / \mathrm{s}\right)$}

There was non-significant bias at 2, 24, 48 and 72 hours respectively for peak moment, mean peak mo- ment and maximum work. Also, the $95 \%$ LoA was similar for peak moment and mean peak moment but higher for maximum work (Table 5).

\subsection{Trunk flexion $\left(15^{\circ} / \mathrm{s}\right)$}

There was a non-significant bias at 2, 24, 48 and 72 hours for maximum and mean peak moment and 
Table 4

Ratio (\%) limits of agreement (LoA) for the baseline value (time 0 hours) compared to 2, 24, 48 and 72 hours during isokinetic contractions of the knee extensors and flexors at angular velocities of 60 and $180^{\circ} / \mathrm{s}(n=10)$. Data are presented as bias $( \pm 95 \%$ LoA). *Denotes significant Bias $(P<0.05)$

\begin{tabular}{|c|c|c|c|c|c|}
\hline Measure & & $2 \mathrm{~h}$ & $24 \mathrm{~h}$ & $48 \mathrm{~h}$ & $72 \mathrm{~h}$ \\
\hline \multirow[t]{3}{*}{ Knee Extension $\left(60^{\circ} / \mathrm{s}\right)$} & Peak Moment (Nm) & $0.5( \pm 15.6)$ & $-1.0( \pm 14.8)$ & $-0.3( \pm 12.9)$ & $2.3( \pm 13.7)$ \\
\hline & Mean Peak Moment (Nm) & $2.1( \pm 11.6)$ & $0.6( \pm 16.0)$ & $3.2( \pm 13.9)$ & $4.2( \pm 15.7)$ \\
\hline & Maximum Work (J) & $3.6( \pm 22.8)$ & $2.3( \pm 20.3)$ & $5.9 *( \pm 16.1)$ & $5.1( \pm 25.2)$ \\
\hline \multirow[t]{3}{*}{ Knee Extension $\left(180^{\circ} / \mathrm{s}\right)$} & Peak Moment (Nm) & $-0.5( \pm 8.3)$ & $1.1( \pm 14.5)$ & $0.8( \pm 6.3)$ & $2.0( \pm 13.3)$ \\
\hline & Mean Peak Moment (Nm) & $-0.4( \pm 12.5)$ & $3.6( \pm 25.6)$ & $3.1( \pm 16.6)$ & $4.8( \pm 25.6)$ \\
\hline & Maximum Work (J) & $3.9( \pm 39.3)$ & $-0.4( \pm 48.1)$ & $2.0( \pm 35.4)$ & $2.3( \pm 40.3)$ \\
\hline \multirow[t]{3}{*}{ Knee Flexion $\left(60^{\circ} / \mathrm{s}\right)$} & Peak Moment (Nm) & $0.3( \pm 21.2)$ & $1.3( \pm 15.4)$ & $3.2( \pm 19.8)$ & $0.9( \pm 22.3)$ \\
\hline & Mean Peak Moment (Nm) & $3.7( \pm 18.8)$ & $3.3( \pm 10.4)$ & $5.0( \pm 18.4)$ & $2.2( \pm 20.7)$ \\
\hline & Maximum Work (J) & $2.7( \pm 31.1)$ & $2.9( \pm 27.1)$ & $5.4( \pm 31.5)$ & $3.4( \pm 34.4)$ \\
\hline \multirow[t]{3}{*}{ Knee Flexion $\left(180^{\circ} / \mathrm{s}\right)$} & Peak Moment (Nm) & $3.7 *( \pm 9.2)$ & $6.4 *( \pm 18.3)$ & $5.1( \pm 21.1)$ & $5.1( \pm 23.9)$ \\
\hline & Mean Peak Moment (Nm) & $2.5( \pm 16.1)$ & $5.4( \pm 17.6)$ & $3.7( \pm 26.6)$ & $4.9( \pm 26.5)$ \\
\hline & Maximum Work (J) & $4.0( \pm 41.9)$ & $9.8( \pm 48.1)$ & $13.3^{*}( \pm 39.9)$ & $10.2( \pm 54.3)$ \\
\hline
\end{tabular}

Table 5

Ratio (\%) limits of agreement (LoA) for the baseline value (time 0 hours) compared to 2, 24, 48 and 72 hours during isokinetic contractions of the trunk extensors and flexors at angular velocities of 15 and $60^{\circ} / \mathrm{s}(n=10)$. Data are presented as bias $( \pm 95 \%$ LoA)

\begin{tabular}{|c|c|c|c|c|c|}
\hline Measure & & $2 \mathrm{~h}$ & $24 \mathrm{~h}$ & $48 \mathrm{~h}$ & $72 \mathrm{~h}$ \\
\hline \multirow[t]{3}{*}{ Trunk Extension $\left(15^{\circ} / \mathrm{s}\right)$} & Peak Moment $(\mathrm{Nm})$ & $-1.8( \pm 16.7)$ & $0.7( \pm 19.7)$ & $-4.1( \pm 19.2)$ & $-6.4( \pm 31.8)$ \\
\hline & Mean Peak Moment (Nm) & $-1.1( \pm 8.3)$ & $-0.3( \pm 18.1)$ & $-4.2( \pm 21.9)$ & $-4.6( \pm 22.1)$ \\
\hline & Maximum Work (J) & $-2.4( \pm 22.0)$ & $-5.0( \pm 29.0)$ & $-2.6( \pm 14.6)$ & $-2.3( \pm 42.9)$ \\
\hline \multirow[t]{3}{*}{ Trunk Extension $\left(60^{\circ} / \mathrm{s}\right)$} & Peak Moment (Nm) & $-1.2( \pm 20.5)$ & $-1.3( \pm 16.3)$ & $-0.3( \pm 21.2)$ & $-4.8( \pm 33.5)$ \\
\hline & Mean Peak Moment (Nm) & $-0.3( \pm 21.8)$ & $-1.6( \pm 15.3)$ & $0.9( \pm 25.5)$ & $-2.8( \pm 35.5)$ \\
\hline & Maximum Work (J) & $-0.5( \pm 45.8)$ & $-8.3( \pm 35.7)$ & $5.5( \pm 64.4)$ & $-11.8( \pm 51.0)$ \\
\hline \multirow[t]{3}{*}{ Trunk Flexion $\left(15^{\circ} / \mathrm{s}\right)$} & Peak Moment (Nm) & $-0.2( \pm 5.5)$ & $1.0( \pm 8.8)$ & $-0.5( \pm 16.6)$ & $-2.6( \pm 17.3)$ \\
\hline & Mean Peak Moment (Nm) & $-0.9( \pm 4.4)$ & $0.7( \pm 10.2)$ & $-2.3( \pm 19.7)$ & $-3.3( \pm 21.5)$ \\
\hline & Maximum Work (J) & $-2.4( \pm 17.1)$ & $-3.8( \pm 23.7)$ & $-5.6( \pm 29.3)$ & $0.3( \pm 47.9)$ \\
\hline \multirow[t]{3}{*}{ Trunk Flexion $\left(60^{\circ} / \mathrm{s}\right)$} & Peak Moment (Nm) & $-1.9( \pm 19.5)$ & $-2.2( \pm 16.6)$ & $-2.1( \pm 23.4)$ & $0.9( \pm 22.9)$ \\
\hline & Mean Peak Moment (Nm) & $-2.7( \pm 20.2)$ & $-2.3( \pm 15.2)$ & $-2.4( \pm 23.1)$ & $-0.6( \pm 29.9)$ \\
\hline & Maximum Work $(\mathrm{J})$ & $1.1( \pm 20.7)$ & $-3.1( \pm 31.4)$ & $-1.9( \pm 42.7)$ & $3.2( \pm 49.7)$ \\
\hline
\end{tabular}

Table 6

Ratio (\%) limits of agreement (LoA) for the baseline value (time 0 hours) compared to 2, 24, 48 and 72 hours during isokinetic contractions of the shoulder extensors and flexors at angular velocities of 60 and $180^{\circ} / \mathrm{s}(n=10)$. Data are presented as bias $( \pm 95 \%$ LoA. *Denotes significant Bias $(P<0.05))$

\begin{tabular}{|c|c|c|c|c|c|}
\hline Measure & & $2 \mathrm{~h}$ & $24 \mathrm{~h}$ & $48 \mathrm{~h}$ & $72 \mathrm{~h}$ \\
\hline \multirow[t]{2}{*}{ Shoulder Extension $\left(60^{\circ} / \mathrm{s}\right)$} & Peak Moment (Nm) & $0.2( \pm 14.7)$ & $1.6( \pm 24.3)$ & $4.2( \pm 27.7)$ & $1.8( \pm 25.3)$ \\
\hline & Mean Peak Moment (Nm) & $0.8( \pm 17.3)$ & $0.9( \pm 18.9)$ & $3.2( \pm 23.9)$ & $2.5( \pm 22.5)$ \\
\hline \multirow[t]{3}{*}{ Shoulder Extension $\left(180^{\circ} / \mathrm{s}\right)$} & Peak Moment (Nm) & $0.3( \pm 18.4)$ & $-3.1( \pm 20.0)$ & $2.4( \pm 23.8)$ & $1.2( \pm 15.7)$ \\
\hline & Mean Peak Moment (Nm) & $2.0( \pm 11.3)$ & $-1.3( \pm 9.2)$ & $3.3( \pm 20.1)$ & $1.8( \pm 11.4)$ \\
\hline & Maximum Work (J) & $2.9( \pm 19.6)$ & $0.8( \pm 19.0)$ & $3.7( \pm 26.5)$ & $1.9( \pm 16.4)$ \\
\hline \multirow{2}{*}{ Shoulder Flexion $\left(60^{\circ} / \mathrm{s}\right)$} & Mean Peak Moment (Nm) & $-2.3( \pm 15.9)$ & $-3.2( \pm 32.8)$ & $-0.2( \pm 25.6)$ & $-3.0( \pm 23.6)$ \\
\hline & Maximum Work (J) & $1.8( \pm 12.9)$ & $2.5( \pm 13.4)$ & $5.7 *( \pm 15.3)$ & $-1.6( \pm 12.3)$ \\
\hline \multirow[t]{3}{*}{ Shoulder Flexion $\left(180^{\circ} / \mathrm{s}\right)$} & Peak Moment (Nm) & $2.1( \pm 18.8)$ & $4.1( \pm 30.5)$ & $-1.6( \pm 23.5)$ & $2.0( \pm 24.4)$ \\
\hline & Mean Peak Moment (Nm) & $4.6( \pm 19.2)$ & $4.7( \pm 30.3)$ & $0.2( \pm 25.6)$ & $5.1( \pm 33.4)$ \\
\hline & Maximum Work (J) & $7.3( \pm 28.9)$ & $10.3( \pm 53.3)$ & $0.8( \pm 33.1)$ & $6.1( \pm 44.2)$ \\
\hline
\end{tabular}

maximum work (Table 5). The 95\% LoA showed that the reproducibility of the measures became slightly poorer over time for all isokinetic parameters (Table 5).
For example, reproducibility of the peak moment compared to baseline was strongest at 2 hours, decreasing at 24 hours $\{1.0( \pm 8.8)\}$ and again at 48 hours $\{-0.5$ 
$( \pm 16.6)\}$ and 72 hours $\{-2.6( \pm 17.3)\}$

\subsection{Trunk flexion $\left(60^{\circ} / \mathrm{s}\right)$}

There was non-significant bias at 2, 24, 48 and 72 hours for peak moment, mean peak moment and maximum work (Table 5). However, compared to trunk flexion $(15 \%)$ there was no systematic change in reproducibility for peak moment or mean peak moment, as shown by $95 \%$ LoA (Table 5). The reproducibility of the maximum work systematically became poorer over time and was lowest at 2 hours $\{1.1( \pm 20.7)\}$, increasing at 24 hours $\{-3.1( \pm 31.4)\}$ and 48 hours $\{-1.9$ $( \pm 42.7)\}$ and peaked at 72 hours $\{3.2( \pm 49.7)\}$.

\subsection{Shoulder extension $\left(60^{\circ} / \mathrm{s}\right)$}

The bias for peak moment, mean peak moment and maximum work was non-significant at 2, 24, 48 and 72 hours (Table 6). Reproducibility (95\% LoA) of measurements was similar for peak moment, mean peak moment and maximum work at 2, 24, 48 and 72 hours (Table 6).

\subsection{Shoulder extension $\left(180^{\circ} / \mathrm{s}\right)$}

There was no significant bias for peak moment, mean peak moment and maximum work (Table 6). The reproducibility was similar for the peak moment, mean peak moment and maximum work at 2, 24, 48 and 72 hours (Table 6).

\subsection{Shoulder flexion $\left(60^{\circ} / \mathrm{s}\right)$}

There was non-significant bias at 2, 24, 48 and 72 hours respectively for peak moment and mean peak moment. However, for maximum work, although there was non-significant bias at 2, 24 and 72 hours respectively $(1.8,2.5,-1.6, P>0.05)$ there was a significant positive bias at 48 hours $(5.7, P=0.040)$. The $95 \%$ LoA was similar for peak moment and mean peak moment but were lower for maximum work (Table 6).

\subsection{Shoulder flexion $\left(180^{\circ} / \mathrm{s}\right)$}

There was non-significant bias at 2, 24, 48 and 72 hours for peak moment, mean peak moment and maximum work (Table 6). Reproducibility of the measurements was similar for peak moment and mean peak moment but generally higher for maximum work, as shown by $95 \%$ LoA (Table 6).

\section{Discussion}

The novelty of this study was that the reproducibility of isokinetic parameters during concentric contractions of extensors and flexors of knee, trunk and shoulder was examined in one testing session over a timescale of three days. This time period is suitable for assessing recovery of neuromuscular function following exercise. For all concentric contractions, fast velocities showed poorer within-day and between-days reproducibility due to the more frequent occurrence of significant mean bias between the baseline and subsequent sessions. Fast velocities also showed consistently poorer reproducibility than slow velocities, indicated by the wider $95 \%$ LoA. This may be due to the relatively shorter rest times in our protocol compared with suggestions for rest time during isokinetic testing [32]. During extension and flexion of knee, trunk and shoulder movements, maximum work showed the greatest variation and peak moment the smallest variation.

Peak torque during knee extension and flexion $\left(60^{\circ} / \mathrm{s}\right)$ in the present study was $198 \pm 40$ and $125 \pm$ $24 \mathrm{Nm}$ at baseline, respectively. Similar values (i.e. 210 and $133 \mathrm{Nm}$ ) were measured during knee extension and flexion in healthy male participants (age 21 to 30 years) [18]. Peak torque during knee extension and flexion $(180 \%$ s) in the present study was $142 \pm 28$ and $93 \pm 13 \mathrm{Nm}$ at baseline, respectively, which is comparable to the 142 and $85 \mathrm{Nm}$ measured during extension and flexion, respectively, in male college track athletes (age 18 to 21 years) [2].

Impellizzeri et al. [20] assessed the reproducibility of peak torque during concentric contractions of the left and right knee extensors and flexors at 60 and $180 \%$ s across 3 test sessions, each separated by $96 \mathrm{~h}$ using intraclass correlation coefficients (i.e. ICC) and LoA. There was minimal difference in reproducibility between the left and the right side. The ICC were high for the left knee extensors at $60 \% \mathrm{~s}(0.95)$ and $180 \% \mathrm{~s}$ $(0.98)$ and the flexors at $60 \%$ s $(0.93)$ and $180 \%$ s $(0.98)$. LoA were calculated using the mean squared error from the two way ANOVA, although statistically valid [3], this method is different from the current study. It does not allow direct comparison of each test to baseline measures or the assessment of how reproducibility may change over time, which was important in addressing the aim of the present study. The LoA of Impellizzeri et al. [20] for peak torque were similar to the present study (Table 4) for knee extension at $60 \%$ s (13.0\%), knee extension at $180 \% \mathrm{~s}(12.5 \%)$, knee flexion at $60 \%$ $(18.6 \%)$ and knee flexion at $180 \%$ s $(13.9 \%)$. These da- 
ta are supported by Nevill and Atkinson [28] who report the ratio $95 \%$ LoA for isokinetic knee extensions and flexions over a range of test velocities. Nevill and Atkinson [28] show a similar 95\% LoA to the present study; for $60 \%$ snee extension (19 vs. $12.9-15.6 \%$ ) and flexion (29 vs. $15.4-22.3 \%$ ) and for $180 \%$ knee extension (16 vs. $6.3-14.5 \%$ ) and flexion (14 vs. 9.2$23.9 \%$ ). The small random changes over time of the LoA in the present study are likely to have been caused by a single outlying individual as illustrated in Figure 2B. The example data in Figure 2B compares baseline and 2 hours for knee extension $180 \%$ s $\{-13.4 *( \pm$ $43.3)\}$. If the outlying participant is removed the mean bias and $95 \%$ LoA are reduced to $\left\{-8.5^{*}( \pm 12.5)\right\}$.

The present study showed slightly poorer reproducibility of work than the peak torque for all contractions. Symons et al. [35] also showed wider 95\% ratio LoA were for work $(39.9 \%)$ compared to peak torque $(32.5 \%)$ during a single test retest of measurements of the knee extensors at $90 \%$ s in older men $(72 \pm$ 5 years) on separate days. Compared to knee extensors $\left(60^{\circ} / \mathrm{s}\right)$ at $24 \mathrm{~h}$ in the present study, Symons et al. [35] data show wider $95 \%$ LoA for peak torque (14.8 vs. 32.5 ) and work (20.3 vs. 39.9). This may be due to the differences between participant characteristics or, as suggested by Symons et al. [35], the absence of a familiarisation session which was used in the present study.

Peak torque during trunk extension $(15 \%)$ in the present study was $288 \pm 38 \mathrm{Nm}$ at baseline, which is comparable to the $250 \mathrm{Nm}$ measured in healthy males (mean age 23 years) [38]. The test velocity was consistent between studies but unlike the present study, Thorstensson and Nilsson [38] measures were taken whilst participants lay on their side. Peak torque during trunk extension $\left(60^{\circ} / \mathrm{s}\right)$ during the present study was $276 \pm 53 \mathrm{Nm}$ at baseline, which is similar to the $255 \mathrm{Nm}$ measured in healthy male participants (age 21 to 30 years) at the same test velocity [18]. Peak torque during trunk flexion $(60 \%)$ in the present study was $296 \pm 34 \mathrm{Nm}$ at baseline which is comparable to the $250 \mathrm{Nm}$ measured in healthy male participants (mean 43 years of age) [37].

In comparison to the knee extensors and flexors, very few studies have assessed reproducibility of contractions of the trunk extensors and flexors using LoA and reproducibility of contractions at $15 \%$ s have not been previously investigated. The LoA for trunk extension and flexion at $60 \%$ in the current study were similar to those previously reported for extension at $30 \%$ s $(\sim 14 \%)$ and $180 \%$ s $(\sim 18 \%)$, trunk flexion $30 \%$ s
( $\sim 20 \%)$ and $180 \%$ s $(\sim 26 \%)$ with the aproximate percentage values calculated from absolute limits presented in original summary data) [14]. However, Nevill and Atkinson [28] reported wider LoA at $60 \%$ for both trunk extension (58\%) and flexion (54\%), the reasons for these differences are not clear as the authors do not describe the procedures or equipment used to collect the data.

Byl and Sadowsky [9] presented high ICC for the peak toque of the trunk extensors over 3 consecutive days at $60 \%$ s (0.97), $90 \%$ s (0.97) and $120 \%$ s (0.95). ICC were poorer for flexion at $60 \% \mathrm{~s}(0.93), 90 \% \mathrm{~s}(0.90)$ and $120 \%$ s $(0.90)$. These findings are in contrast to the findings of the current study which showed that the reproducibility of peak torque for trunk extension and flexion was similar for both 15 and $60 \%$ s. Byl and Sadowsky [9] present lower ICC for work compared to peak torque during extension and flexion across all test velocities. These findings support the current study which showed poorer reproducibility for work compared to peak torque for both extension and flexion at 15 and $60 \%$ s. The present study showed reproducibility of peak torque to be similar for trunk extension at 15 and $60 \%$, but stronger reproducibility during for flexion at $15 \%$ s compared to $60 \%$ s (Table 5). Previous studies have shown weaker reproducibility at higher test speeds for both extension and flexion of the trunk, however test velocities were higher (60 to $180 \%$ s) and different dynamometers were used [14,24].

Peak torque during shoulder extension $(60 \%)$ in the present study was $101 \pm 20 \mathrm{Nm}$ at baseline, which is between the $85 \mathrm{Nm}$ and $118 \mathrm{Nm}$ measured in nonathletic healthy males of similar age [12,34]. Similarly, peak torque during shoulder extension $(180 \%)$ in the present study was $85 \pm 15 \mathrm{Nm}$ at baseline, which is between the $73 \mathrm{Nm}$ and $103 \mathrm{Nm}$ measured in nonathletic healthy male participants of similar age [12, 34]. Peak torque during shoulder flexion $(60 \% \mathrm{~s})$ in the present study was $76 \pm 13 \mathrm{Nm}$ at baseline, which is comparable to the $67 \mathrm{Nm}$ measured in non-athletic healthy males (age 21 to 41 years) [12]. Peak torque during shoulder flexion $\left(180^{\circ} / \mathrm{s}\right)$ in the present study was $54 \pm 9 \mathrm{Nm}$ at baseline, which is comparable to the $54 \mathrm{Nm}$ measured in non-athletic healthy males (age 22 to 30 years) [34].

Reproducibility of shoulder extension and flexion using isokinetic dynamometry has not been previously assessed using the LoA method. Orri and Darden [30] examined the test-retest reproducibility of shoulder extension and flexion $(60 \%$ s) across $3-5$ days. ICC showed strong reproducibility for extension of the left (0.94) 
and right (0.98) shoulder and flexion of the left (0.95) and right (0.95) shoulder. However, Mayer et al. [27] examined the reproducibility of shoulder extension and flexion at $60,180,240,300 \%$ after a 14 day interval. The reproducibility between measurements was deemed to be low and was similar for shoulder extension $(16.3 \%)$ and flexion $(17.2 \%)$. These findings differ from the current study as reproducibility of shoulder extension was slightly stronger than flexion. This may have been due to differences in the participant population, test velocity or time between tests [21].

Timm et al. [39] assessed the mechanical and physiological reproducibility of three dynamometers to assess the knee extensors and flexors (Biodex, Cybex 340, Merac System) and trunk extensors and flexors (Cybex TEF, Cybex Torso, Cybex Liftask) over a range of test velocities (60 to $500 \%$ s). Mechanical reproducibility was measured by attaching a standardised loaded input shaft to the dynamometer arm and allowing the arm to move through a $90^{\circ}$ range of motion 5 times. Physiological reproducibility was assessed by participants ( 86 males, 86 females, age 16-34 years) completing 2 sets of 5 maximal contractions separated by 48 hours. Mechanical test-retest reproducibility (assessed by ICC) of the peak torque assessed on the Cybex 340 was very strong at $60 \%$ s (1.000) and $180 \%$ s (1.000). The ICC of the physiological reproducibility of the peak torque were also strong at $60 \%$ for the knee extensors (0.999) and flexors (0.999) and at $180 \%$ s for the knee extensors (0.999) and flexors (0.979). A similar pattern was observed for the other dynamometers and during trunk extension and flexion. The authors concluded that differences tended to be found in the physiological testing therefore the source of variability when using isokinetic dynamometry is due to the participants rather than the dynamometers.

In summary, combined testing of knee, shoulder and trunk muscles during maximal concentric slow and fast concentric contractions provides moment values that are comparable to studies examining a single muscle group. Peak moment is the most reliable isokinetic parameter for knee, trunk and shoulder extensors to assess in one testing session. In addition, reproducibility of this parameter is maintained over a 3-day time period. However, when relatively large variability of isokinetic parameters was detected, it was likely due to individual subjects rather than the dynamometer, therefore adequate familiarisation and coaching is required to maintain reproducibility. Caution is needed to interpret results at fast velocities due to the poorer reproducibility between tests over time. The implication of the present study is that the assessment of the recovery of isokinetic function at fast and slow concentric contractions of knee, trunk and shoulder extensors and flexors in the days following exercise may require peak moment to be the preferred isokinetic parameter.

\section{References}

[1] H.J. Appell, J.M. Soares and J.A. Duarte, Exercise, muscle damage and fatigue, Sports Medicine 13 (1992), 108-115.

[2] L. Appen and P.W. Duncan, Strength relationship of the knee musculature: effects of gravity and sport, Journal of Orthopaedic and Sports Physical Therapy 7 (1986), 232-235.

[3] G. Atkinson and A.M. Nevill, Statistical methods for assessing measurement error (reliability) in variables relevant to sports medicine, Sports Medicine 26 (1998), 217-238.

[4] D.J. Bentley, P.A. Smith, A.J. Davie and S. Zhou, Muscle activation of the knee extensors following high intensity endurance exercise in cyclists, European Journal of Applied Physiology 81 (2000), 297-302.

[5] J.M. Bland and D.G. Altman, Statistical methods for assessing agreement between two methods of clinical measurement, Biochimica Clinica 11 (1987), 399-404.

[6] J.M. Bland and D.G. Altman, Comparing methods of measurement: why plotting difference against standard method is misleading, Lancet 21 (1995), 1085-1087.

[7] J.M. Bland and D.G. Altman, Applying the right statistics: analyses of measurement studies, Ultrasound in Obstetrics and Gynecology 22 (2003), 85-93.

[8] J.M. Bland and D.G. Altman, Agreement between methods of measurement with multiple observations per individual, Journal of Biopharmaceutical Statistics 17 (2007), 571-582.

[9] N.N. Byl and H.S. Sadowsky, Intersite reliability of repeated isokinetic measurements: Cybex back systems including trunk rotation, trunk extension-flexion, and liftask, Isokinetics and Exercise Science 3 (1993), 139-147.

[10] C. Byrne and R. Eston, The effect of exercise-induced muscle damage on isometric and dynamic knee extensor strength and vertical jump performance, Journal of Sports Sciences $\mathbf{2 0}$ (2002), 417-425.

[11] C. Byrne, C. Twist and R. Eston, Neuromuscular function after exercise-induced muscle damage: theoretical and applied implications, Sports Medicine 34 (2004), 49-69.

[12] T.D. Cahalan, M.E. Johnson and E.Y. Chao, Shoulder strength analysis using the Cybex II isokinetic dynamometer, Clinical and Orthopaedic Related Research 271 (1991), 249-257.

[13] E.N. Corlett and R.P. Bishop, A technique for assessing postural discomfort, Ergonomics 19 (1976), 175-182.

[14] E. Derviševic, V. Hadžic and H. Burger, Reproducibility of trunk isokinetic strength findings in healthy individuals, Isokinetics and Exercise Science 15 (2007), 99-109.

[15] Z. Dvir, Isokinetics. 1st ed. New York: Churchill Livingstone, 1995.

[16] R.G. Eston, S. Finney, S. Baker and V. Baltzopoulos, Muscle tenderness and peak torque changes after downhill running following a prior bout of isokinetic eccentric exercise, Journal of Sports Sciences 14 (1996), 291-299.

[17] M. Fillyaw, T. Bevins and L. Fernandez, Importance of correcting isokinetic peak torque for the effect of gravity when calculating knee flexor to extensor muscle ratios, Physical Therapy 66 (1986), 23-31. 
[18] P.S. Freedson, T.B. Gilliam, T. Mahoney, A.F. Maliszewski and K. Kastango, Industrial torque levels by age group and gender, Isokinetics and Exercise Science 3 (1993), 34-42.

[19] E. Gauche, R. Lepers, G. Rabita, J.M. Leveque, D. Bishop, J. Brisswalter and C. Hausswirth, Vitamin and mineral supplementation and neuromuscular recovery after a running race, Medicine and Science in Sports and Exercise 38 (2006), 2110-2117.

[20] F.M. Impellizzeri, M. Bizzini, E. Rampinini, F. Cereda and N.A. Maffiuletti, Reliability of isokinetic strength imbalance ratios measured using the Cybex NORM dynamometer, Clinical Physiological and Functional Imaging 28 (2008), 113119 .

[21] J.L. Keating and T.A. Matyas, The influence of subject and test design on dynamometric measurements of extremity muscles, Physical Therapy 76 (1996), 866-889.

[22] J.J. Knapik and M.U. Ramos, Isokinetic and isometric torque relationships in the human body, Archives of Physical Medicine and Rehabilitation 61 (1980), 64-67.

[23] R. Lepers, G.Y. Millet and N.A. Maffiuletti, Effect of cycling cadence on contractile and neural properties of knee extensors, Medicine and Science in Sports and Exercise 33 (2001), 18821888.

[24] O.R. Madsen, Trunk extensor and flexor strength measured by the Cybex 6000 dynamometer. Assessment of short-term and long-term reproducibility of several strength variables, Spine 21 (1996), 2770-2776.

[25] V. Martin, G.Y. Millet, G. Lattier and L. Perrod, Effects of recovery modes after knee extensor muscles eccentric contractions, Medicine and Science in Sports and Exercise 36 (2004), 1907-1915.

[26] F. Mayer, T. Horstmann, U. Kranenberg, K. Rocker and H.H. Dickhuth, Reproducibility of isokinetic peak torque and angle at peak torque in the shoulder joint, International Journal of Sports Medicine 15(Suppl 1) (1994), S26-S31.

[27] G.Y. Millet and R. Lepers, Alterations of neuromuscular function after prolonged running, cycling and skiing exercises, Sports Medicine 34 (2004), 105-116.

[28] A.M. Nevill and G. Atkinson, Assessing agreement between measurements recorded on a ratio scale in sports medicine and sports science, British Journal of Sports Medicine 31 (1997), 314-318.

[29] C. Nottle and K. Nosaka, The magnitude of muscle damage induced by downhill backward walking, Journal of Science and Medicine in Sport 8 (2005), 264-273.

[30] J.C. Orri and G.F. Darden, Technical report: Reliability and validity of the iSAM 9000 isokinetic dynamometer, Journal of Strength and Conditioning Research 22 (2008), 310-317.

[31] D. Paddon-Jones, M. Muthalib and D. Jenkins, The effects of a repeated bout of eccentric exercise on indices of muscle damage and delayed onset muscle soreness, Journal of Science and Medicine in Sport 3 (2000), 35-43.

[32] A.C. Parcell, R.D. Sawyer, V.A. Tricoli and T.D. Chinevere, Minimum rest period for strength recovery during a common isokinetic testing protocol, Medicine and Science in Sports and Exercise 34 (2002), 1018-1022.

[33] D.H. Perrin, Isokinetic Exercise and Assessment, Champaign: Human Kinetics, 1993.

[34] A.Shklar and Z. Dvir, Isokinetic strength relationships in shoulder muscles, Clinical Biomechanics 10 (1995), 369-373.

[35] T.B. Symons, A.A. Vandervoort, C.L. Rice, T.J. Overend and G.D. Marsh, Reliability of a single-session isokinetic and isometric strength measurement protocol in older men, The Journals of Gerontology Series A: Biological Sciences and Medical Sciences 60 (2005), 114-119.

[36] J.C. Tee, A.N. Bosch and M.I. Lambert, Metabolic consequences of exercise-induced muscle damage, Sports Medicine 37 (2007), 827-836.

[37] N.N. Thompson, J.A. Gould, G.J. Davies, D.E. Ross and S. Price, Descriptive measures of isokinetic trunk testing, Journal of Orthopaedic and Sports Physical Therapy 7 (1985), 43-49.

[38] A. Thorstensson and J. Nilsson, Trunk muscle strength during constant velocity movements, Scandinavian Journal of Rehabilitation Medicine 14 (1982), 61-68.

[39] K.E. Timm, P. Gennrich, R. Burns and D. Fyke, The mechanical and physiological performance reliability of selected isokinetic dynamometers, Isokinetics and Exercise Science 2 (1992), 182-190.

[40] G.L. Warren, C.P. Ingalls, D.A. Lowe and R.B. Armstrong, What mechanisms contribute to the strength loss that occurs during and in the recovery from skeletal muscle injury?, Journal of Orthopaedic and Sports Physical Therapy 32 (2002), 58-64.

[41] M.R. Wilhite, E.R. Cohen and S.C. Wilhite, Reliability of concentric and eccentric measurements of quadriceps performance using the kin-com dynamometer: the effect of testing order for three different speeds, Journal of Orthopaedic \& Sports Physical Therapy 15 (1992), 175-182. 
Copyright of Isokinetics \& Exercise Science is the property of IOS Press and its content may not be copied or emailed to multiple sites or posted to a listserv without the copyright holder's express written permission.

However, users may print, download, or email articles for individual use. 